Artículo original

\title{
LA NUEVA GESTIÓN PÚBLICA Y LA APLICACIÓN DE INDICADORES DE GESTIÓN EN LAS MUNICIPALIDADES PROVINCIALES DE LAMACRO REGIÓN SUR, 2014.
}

The new public management and the application of management indicators in the provincial municipalities of the southern macro region, 2014.

\author{
Elberth Hernán Samalvides Márquez ${ }^{1}$
}

\begin{abstract}
RESUMEN
La investigación tuvo el propósito de determinar la influencia de la nueva Gestión Pública en la aplicación de los indicadores de gestión en las Municipalidades Provinciales de la Macro Región Sur 2014. Investigación de tipo básica a 51 gerentes y funcionarios de los gobiernos municipales provinciales de la Macro Región sur del Perú: Apurímac, Arequipa, Moquegua, Tacna, Cusco, Madre de Dios y Puno. Se determinó que la Nueva Gestión Pública influye significativamente en la aplicación de los indicadores de Gestión de Municipalidades de la Macro Región Sur 2014 y la evaluación de la nueva Gestión Pública en las Municipalidades Provinciales de la Macro Región Sur 2014, es inadecuada.
\end{abstract}

Palabras Clave: Gestión Pública, Municipalidades.

ABSTRACT: The research was aimed at determining the influence of the new Public Management in the application of management indicators in the Provincial Municipalities of the Macro Region South 2014. Basic type research to 51 managers and officials of the provincial municipal governments of The southern Region of Peru: Apurimac, Arequipa, Moquegua, Tacna, Cusco, Madre de Dios and Puno. It was determined that: The New Public Management has a significant influence on the application of the indicators of Municipal Management of the Macro Region South 2014 and the evaluation of the new Public Management in the Provincial Municipalities of Macro Region South 2014 is inadequate.

KeyWords: Public Management, Municipalities.

\section{INTRODUCCIÓN}

Durante las tres últimas décadas la reforma de la gestión pública ha formado parte de la agenda política del Perú, aunque con resultados aún insuficientes en el logro de la denominada economía del bienestar. El sistema de municipalidades peruanas está entrando en una nueva etapa, signada por la necesidad de responder ante

\footnotetext{
Doctor en administración

Coordinador de la escuela Profesional de Administración Univ. San Martin de Porres. Docente de Postgrado en la UNIV Catolica Santa María.
}

poblaciones cada vez más ilustradas en los asuntos públicos. Hablar de buen gobierno y gobernabilidad en los municipios es un tema muy complejo en la realidad peruana, pero a la vez, se trata de un tema ineludible. En la investigación, abordamos estos temas para buscar una disquisición académica, y adentrarnos en una preocupación muy sentida entre los gobiernos locales y la ciudadanía. Implementar indicadores para medir la gestión del buen gobierno y la gobernabilidad municipal tiene una utilidad inmediata y justificada. No obstante siendo tan relevante la construcción de una base de datos, por sus posibilidades de emplearse estratégicamente en la gestión local, es necesario señalar que no toda la información reside al interior de la municipalidad. La presente investigación medirá la influencia de la nueva Gestión Pública en la aplicación de los indicadores de gestión en las municipalidades provinciales de la Macro Región sur 2014.

Los municipios en el Perú presentan limitaciones en la aplicación de los indicadores en la gestión pública, lo que no permite una mayor eficiencia, eficacia y economía en el uso de los fondos públicos. Se actúa en un medio marcado siempre por tensiones entre quienes tratan de atender las necesidades comunes y otros que buscan la obtención de beneficios particulares. A nivel nacional la implementación de la Nueva Gestión Pública, no ha logrado la modernización de la gestión pública en los municipios provinciales y distritales del país. A la fecha, todavía persisten deficiencias como ausencia de un sistema eficiente de planeamiento, deficiente diseño de la estructura de organización y funciones, inadecuados procesos de producción de bienes y servicios públicos, inadecuada política y gestión de recursos humanos, limitada evaluación de resultados e impactos y carencia de sistemas y metas de gestión. A nivel de la macro región sur que incluye Apurímac, Arequipa, Moquegua, Tacna, Cusco, Madre de Dios y Puno la implementación de la nueva gestión pública reproduce los problemas 0 limitaciones anteriormente señaladas. La investigación tiene importancia teórica porque está orientada a lograr un mayor conocimiento y comportamiento de las variables en estudio, gestión pública, indicadores de gestión así mismo los factores que han limitado su éxito. 
La administración es un conjunto ordenado y sistematizado de principios, técnicas y prácticas que tiene como finalidad perseguir los objetivos de una organización y obtener resultados con la mayor eficiencia, eficacia y congruencia a través de la óptima coordinación y aprovechamiento del personal y los recursos técnicos, materiales y financieros. En el ámbito municipal, la administración se refiere a las acciones relacionadas con el manejo adecuado y eficiente de los recursos humanos, materiales y financieros. Castillo en el 2004(Castillo, J., 2004a) en Perú, concluye que la reingeniería en la gestión de los gobiernos locales permite optimizar la prestación de los servicios municipales en beneficio de los ciudadanos, racionalizando procesos, integrando las diferentes áreas funcionales a través de los sistemas de información gerencial y estableciendo canales de comunicación directa con los vecinos. La descentralización política, administrativa y económica, permitirá a los gobiernos regionales y locales en particular a autoadministrarse. La prestación de los servicios municipales a la comunidad que brindan los gobiernos locales, deben estar basados en los principios de igualdad, permanencia 0 de continuidad, adecuación, generalidad, legalidad, persistencia y adaptación.

Es sabido que existe ausencia de capacitación por parte de las entidades que desarrollan el Sistema de información de Administración Financiera (SIAF) a cada gerente de los gobiernos locales y sus subordinados.(Mendighttti C. A., 2012) . Cuanto mayor es el grado de implicación de los agentes interesados en el proceso de diseño de los sistemas de indicadores mayor es la probabilidad de éxito de la iniciativa(Erkizia O., Agustín, 2012). Es necesario la necesidad de discutir -teórica y prácticamente- los desafíos que deben ser asumidos por la gestión pública en el manejo de la elaboración presupuestal por resultados y la rendición de cuentas, especialmente considerándolas herramientas útiles en el proceso de asegurar un «buen gobierno(Franciskovic, J., 2013). Existe un nivel intermedio hacia el aprendizaje organizacional y que hay condiciones que requieren mejorar para lograr organizaciones que aprendan en el sector público (Encinas O. F., 2014). Debido a los múltiples factores que deben ser evaluados, es difícil de conseguir con una única solución mejorar la formulación de políticas y la implementación administrativa. Todas las acciones deben ser tomadas en grupos y por un corto período de tiempo(Matei, A. \& Antonie, C, 2014). El Estado en su doble carácter de gobierno y administrador cumple sus fines, competencias y funciones en sus órganos jurídicos que forman una estructura especial y un conjunto de técnicas y procedimientos que lo ponen en marcha (Torres, L., 2002).
Las expresiones gestionar y administrar aparecen como sinónimos, similares, de uso indistinto. Sin embargo en la legislación peruana estos conceptos aparecen diferenciados(Aibar, G., 2003). El modelo burocrático o weberiano, fue el paradigma inspirador de todo movimiento internacional de reforma administrativa de los años 50 y 60 , fue el modelo que inspiró tras la segunda guerra mundial, característico del Estado Social(Díaz, D. \& Xifrá, J. V., 2005). Ese modelo burocrático buscaba la eficiencia, entendia a ella como su paradigma. En esa concepción para lograr eficiencia se despersonaliza la gestión, las personas tienen como fundamento el cumplimiento de lo que la institucionalidad formal les define(Díaz, D. \& Xifrá, J. V., 2005). Los funcionarios cumplen estrictamente lo establecido por el contenido del cargo y ejecutan lo que las normas establecen sin discusión alguna. En todo caso, la gestión de los servidores públicos debía guiarse por la obligación y no por la misión. De esta forma, el modelo Weberiano efectuaba la distinción entre un experto y un tomador de decisiones. El experto (científico) ejecutaba lo que el tomador de decisiones (político) establecía en función de su lectura de la realidad. Separaba, consiguientemente, la administración de la política. El modelo post burocrático, enfatiza el beneficio que la sociedad percibe del accionar público. Los resultados se miden en términos de cambios de distinta escala que se dan en el entorno interno y externo - de la organización pública (Díaz, D. \& Xifrá, J. V., 2005)

La nueva gestión pública busca satisfacer las necesidades de los ciudadanos a través de una gestión pública eficiente y eficaz. Para este enfoque, es imperativo el desarrollo de servicios de mayor calidad en un marco de sistemas de control que permitan transparencia en los procesos de elección de planes y resultados, así como en los de participación ciudadana. Es el paradigma donde se inscriben los distintos procesos de cambio en la organización y gestión de las administraciones públicas. En la nueva gestión pública los directivos públicos elegidos deben resolver la ambigüedad, la incertidumbre y el conflicto alrededor de las políticas públicas en un lapso determinado y las funciones de formulación de políticas y las de operaciones deberían asignarse a organizaciones diferentes. Traslada obligaciones, autoridad y responsabilidad a los niveles más cercanos al ciudadano (González I. \& M., Ricardo, 2010). Los Sistemas Administrativos tienen por finalidad regular la utilización de los recursos en las entidades de la administración pública, promoviendo la eficacia y eficiencia en su uso (Hintze, J., 2003). En el marco del proceso de descentralización, el ejercicio del poder entre el Gobierno Nacional, los gobiernos regionales y locales debe ser equilibrado, de cooperación y 
colaboración mutua (Armijo, M., 2011). Actualmente existe una gran oportunidad para los gobiernos regionales y las municipalidades para que puedan mejorar y optimizar sus instituciones, sin embargo hay pocas iniciativas al respecto, porque el desafío es de gran envergadura y complejo (Pérez L. \& Ortiz D., 2011).

Las municipalidades, provinciales 0 distritales, pueden crear organismos descentralizados para el mejor cumplimiento de sus competencias. La transferencia de funciones a estos organismos puede asumir diferentes formas (Pérez L. \& Ortiz D., 2011). De acuerdo a la Constitución Política las municipalidades son órganos de gobierno local, con autonomía política, económica y administrativa en los asuntos de su competencia. La planificación estratégica global unida a la ordenación del territorio vía Planes Generales es el mejor fomento de la participación ciudadana; la defensa de lo público. La Secretaría de Gestión Pública de la Presidencia del Consejo de Ministros (SGP está impulsando un proceso de Modernización de la Gestión Pública, enfocada en resultados y que rinda cuentas a los ciudadanos (Castillo, J., 2004b).

\section{OBJETIVOS}

a) Identificar las características de la nueva Administración de la Gestión Pública en los Municipios Provinciales de la Macro Región Sur 2014.

b) Analizar la aplicación de los indicadores de gestión en las Municipalidades Provinciales de la Macro Región Sur 2014.

\section{METODOLOGÍA.}

La investigación es de tipo básica y naturaleza fáctica, de carácter transversal. Nivel descriptivo causal explicativo. No experimental, porque no se han manipulado las variables. El ámbito espacial de la investigación estuvo representada por los gobiernos locales de Apurímac, Arequipa, Moquegua, Tacna, Cusco, Madre de Dios, y Puno en el año 2014. La muestra estuvo constituida por 51 funcionarios de las Municipales descritas. Se utilizó técnicas documentales, las cuales permitieron la revisión de normas, leyes, libros, revistas, tesis, artículos, informes y monografías. El cuestionario fue el instrumento de campo. El instrumento de medición del cuestionario fue validado por expertos y sometido a la prueba de confiabilidad del Alfa de Cronbach $(0,786$ para el cuestionario Indicadores de Gestión y 0,807 para el cuestionario Nueva Gestión Pública).

\section{RESULTADOS}

Podemos observar (tabla 01), que en el año 2009 el $17.65 \%$ de las municipalidades gastaron menos de lo que presupuestaron lo cual implica que esa diferencia de dinero tuvo que ser revertido al gobierno central. Este porcentaje de municipalidades se incrementó hasta alcanzar el $27.45 \%$ en el año 2011 y el $19.61 \%$ en el 2012 , Para el año siguiente este porcentaje volvió a su nivel inicial del periodo $(17.65 \%)$.

Tabla 1: Municipalidades con presupuesto mayor a lo ejecutado

\begin{tabular}{ccc}
\hline Año & \multicolumn{2}{c}{$\begin{array}{c}\text { Municipalidades Provinciales de } \\
\text { la Macro Región Sur }\end{array}$} \\
\cline { 2 - 3 } & $\mathbf{N}$ & $\mathbf{\%}$ \\
\hline $\mathbf{2 0 0 9}$ & 9 & 17.65 \\
$\mathbf{2 0 1 0}$ & 9 & 17.65 \\
$\mathbf{2 0 1 1}$ & 14 & 27.45 \\
$\mathbf{2 0 1 2}$ & 10 & 19.61 \\
$\mathbf{2 0 1 3}$ & 9 & 17.65 \\
TOTAL & $\mathbf{5 1}$ & $\mathbf{1 0 0 . 0 0}$ \\
\hline
\end{tabular}

Respecto al cumplimiento de los instrumentos de gestión según normativa vigente vemos que en su mayoría el nivel en esta dimensión tiende a un nivel nulo y bajo (tabla 2). 
Tabla 2: Cumplimiento de los instrumentos de gestión según normativa

\begin{tabular}{lcccccc}
\hline $\begin{array}{l}\text { Cumplimiento de } \\
\text { Instrumentos }\end{array}$ & $\mathbf{n}$ & Nulo & Bajo & Medio & Alto & $\begin{array}{c}\text { Muy } \\
\text { alto }\end{array}$ \\
\hline $\begin{array}{l}\text { Plan de desarrollo } \\
\text { Institucional }\end{array}$ & 51 & 15.69 & 50.98 & 7.84 & 5.88 & 19.61 \\
$\begin{array}{l}\text { Plan Estratégico y } \\
\text { Desarrollo económico }\end{array}$ & 51 & 17.65 & 49.02 & 31.37 & 0.00 & 1.96 \\
$\begin{array}{l}\text { Plan Operativo } \\
\text { Presupuesto }\end{array}$ & 51 & 5.88 & 62.75 & 25.49 & 5.88 & 0.00 \\
$\begin{array}{l}\text { Participativo } \\
\begin{array}{l}\text { Instrumentos } \\
\text { organizacionales }\end{array}\end{array}$ & 51 & 7.84 & 64.71 & 23.53 & 1.96 & 1.96 \\
Portal Institucional & 51 & 13.73 & 45.10 & 23.53 & 13.73 & 3.92 \\
\hline
\end{tabular}

Respecto a "Nueva gestión Pública" (tabla 3) los aspectos más desfavorables son "Reorganización e implicación de la función pública en la gestión y reforma" $(70.59 \%)$, "Desregulación" (66,67\%) y "Énfasis en las responsabilidad de gestión" (66.67\%), Introducción de la competencia y el mercado" y Métodos de evaluación y técnicas de gestión donde corresponde a un $41.18 \%$.

Tabla 3: Nueva gestión pública

\begin{tabular}{|c|c|c|c|c|c|c|}
\hline Nueva Gestión Publica & $\mathbf{n}$ & Nulo & Bajo & Medio & Alto & $\begin{array}{l}\text { Muy } \\
\text { alto }\end{array}$ \\
\hline Desregulación & 51 & 15.69 & 50.98 & 7.84 & 5.88 & 19.61 \\
\hline Gestión orientada hacia el cliente & 51 & 19.61 & 45.10 & 21.57 & 9.80 & 3.92 \\
\hline $\begin{array}{l}\text { Énfasis en las responsabilidad } \\
\text { de Gestión }\end{array}$ & 51 & 15.69 & 50.98 & 7.84 & 5.88 & 19.61 \\
\hline $\begin{array}{l}\text { Introducción de la competencia y } \\
\text { el mercado }\end{array}$ & 51 & 15.69 & 50.98 & 7.84 & 5.88 & 19.61 \\
\hline $\begin{array}{l}\text { Métodos de evaluación y Técnica } \\
\text { de Gestión }\end{array}$ & 51 & 15.69 & 25.49 & 39.22 & 13.73 & 5.88 \\
\hline Descentralización & 51 & 27.45 & 37.25 & 19.61 & 1.96 & 13.73 \\
\hline $\begin{array}{l}\text { Reorganización e implicancia de } \\
\text { la función pública en la gestión } \\
\text { y reformas }\end{array}$ & 51 & 17.65 & 52.94 & 29.41 & 0.00 & 0.00 \\
\hline
\end{tabular}

Se evaluaron quienes eran los "Usuarios de la Información municipal", encontrándose que el ítem más desfavorable era "contraloría general" (72.55\%"), "Políticos" y "Otros municipios" (70.58\%) y "consejero municipal" (49.02\%.). Frente a los indicadores de gestión, los más desfavorables fueron los "Indicadores de Eficiencia" e "Indicadores de eficacia" (66.67\%). La implementación de indicadores de gestión, mostraron una proporción altamente desfavorable los aspectos de "Presupuesto por resultados" (82.35\%) "Sistema de indicadores" (78.44\%) y en menor proporción "Contabilidad de costos" (35.29\%). Los que menos participan en la elaboración de dichos indicadores son Empleados (82.36\%), Instituciones externas $(74.51 \%)$ y Proveedores $(70.59 \%)$. Muestran menor proporción de negatividad los Ciudadanos (39.22\%), Funcionarios $(27.45 \%)$ y Regidores $(23.53 \%)$.

Según la dimensión "Usuarios de los resultados"; Ios que muestran nula o bajo nivel son la "Asociación de las Municipalidades del Perú" (74.51\%) , "Ciudadanos" (68.63\%) e "Instituciones Académicas" $(60.78 \%)$. Tuvieron un nivel medio sólo "Regidores" (43.14\%).

Observamos también una escasa transparencia de la información municipal en cuanto a indicadores de 
gestión y con qué periodicidad lo realiza, se observa que el $9.80 \%$ de ellas lo hace mensualmente, $5.88 \%$ bimestral mente, $39.22 \%$ semestralmente, $13.73 \%$ en forma anual, y el $31.37 \%$ de municipalidades no difunde sus indicadores de gestión (tabla 4).

Tabla 4: La periodicidad de la difusión de los indicadores de gestión

\begin{tabular}{ccc}
\hline Periodicidad & \multicolumn{2}{c}{$\begin{array}{c}\text { Municipalidades Provinciales } \\
\text { de la Macro Región Sur }\end{array}$} \\
\cline { 2 - 3 } & $\mathrm{n}$ & $\%$ \\
\hline Mensual & 5 & 9.80 \\
Bimestral & 3 & 5.88 \\
Semestral & 20 & 39.22 \\
Anual & 7 & 13.73 \\
No realiza & 16 & 31.37 \\
TOTAL & 51 & 100.00 \\
\hline
\end{tabular}

Según la escala de valoración ensayada en la presente investigación, un poco más de la tercera parte de los funcionarios y gerentes de la municipalidad opinan que la nueva gestión pública tiene un nivel de "Mala" (62.75\%), un poco más de la cuarta parte opinan que es "Regular" (29.41\%). Ninguno de los funcionarios sujetos de estudio valoró que la nueva gestión Pública es "buena" o "Muy buena" (Tabla 05). Asimismo respecto a los indicadores de Gestión, es considerado como "Malo" en el $72.55 \%$. Un poco más de la quinta parte opinan que es "Regular" (21.57\%) y una minoría opina que es "Muy mala" (3.92\%). En mucha menor proporción opinan que el nivel de los Indicadores de gestión es "buena" (1.96\%) (Tabla 6).

Tabla 5: Nivel de la Nueva Gestión Pública

\begin{tabular}{lcc}
\hline & \multicolumn{2}{c}{ Municipalidades Macro Región Sur } \\
\cline { 2 - 3 } & $\mathrm{N}$ & $\%$ \\
\hline MUY MALA & 4 & 7,84 \\
MALA & 32 & 72,75 \\
REGULAR & 15 & 19,41 \\
BUENA & 0 & 0,00 \\
MUY BUENA & 0 & 0,00 \\
\hline \multicolumn{1}{c}{ Total } & 51 & 100,00 \\
\hline \multicolumn{2}{c}{0.000}
\end{tabular}

Tabla 6: Nivel de Indicadores de Gestión

\begin{tabular}{lcc}
\hline & \multicolumn{3}{c}{ Municipalidades Macro Región } \\
& N & Sur \\
& 2 & $\%$ \\
\hline MUY MALA & 37 & 3,92 \\
MALA & 11 & 72,55 \\
REGULAR & 1 & 21,57 \\
BUENA & 0 & 1,96 \\
MUY BUENA & 51 & 0.00 \\
\hline \multicolumn{1}{c}{ Total } & p: 0.000 & 100,0 \\
\hline
\end{tabular}

\section{DISCUSIÓN}

González, (2000), sostiene que la Nueva Gestión Pública busca satisfacer las necesidades de los ciudadanos a través de una gestión pública eficiente y eficaz. Para este enfoque, es imperativo el desarrollo de servicios de mayor calidad en un marco de sistemas de control que permitan transparencia 
en los procesos de elección de planes y resultados, así como en los de participación ciudadana.

Se ha comprobado que en algunas Municipalidades Provinciales se efectúan una deficiente elaboración de los presupuestos, ya que se gasta menos de lo que se presupuestó como gastos, lo cual implica que algunos recursos se regresen o reviertan al Gobierno Central, (en promedio en casi el $20 \%$ de la Municipalidades Provinciales para el periodo de estudio, presentan estas situaciones). Quizás una de las causas de que ello suceda se deba a que el nivel de formación del responsable de la Gestión económica y financiera es muy pobre. Esta situación empeora cuando se trata de los municipios distritales, por lo que hace necesario tomar algunas medidas urgentes al respecto. En lo referente a las actividades y/o servicios que deben prestar la Municipalidades, se tiene que casi el $67 \%$ lo hace por gestión indirecta. En cuanto al cumplimiento de los instrumentos de gestión municipal sólo el $25 \%$ (en promedio) de las municipalidades provinciales de la macro región sur cumplen con ello de una manera aceptable, solo el $6 \%$ de ellas lo hace de forma muy precisa. Sobre el posicionamiento de las municipalidades provinciales según la función que cumplen a nivel de gestión, se tiene que sólo aproximadamente el $22 \%$ (en promedio) cumplen de forma eficiente con los postulados de la Nueva Gestión Pública. Solo el $10 \%$ de municipalidades provinciales han implementado instrumentos de gestión de una manera aceptable; el $15 \%$ de municipalidades desarrolla e implementa indicadores de gestión.

En cuanto al uso de estos indicadores de gestión municipal sólo en el $15 \%$ de las municipalidades esto se realiza. En cuanto a la transparencia municipal se encontró que el $25 \%$ de municipalidades no cumple con el portal institucional y el $30 \%$ de ellas no difunde los indicadores de gestión.

Ante la crítica situación encontrada en las municipalidades provinciales de la macro región sur, la cual se agudiza cuando se trata de municipios distritales, se hace necesaria la implementación urgente de un Plan de Desarrollo Municipal, para que de esta forma estas instituciones cumplan con los objetivos para lo que fueron creadas. Una de las estrategias para lograr este cambio de rumbo se articula a través del desarrollo local porque, las repercusiones de la globalización afectan a las vidas y aspiraciones de la gente en las comunidades en las que ésta vive y trabaja. Una mayor participación de la sociedad civil en la toma de decisiones a través, por ejemplo, del sistema de presupuestos participativos, constituye el caldo de cultivo necesario para iniciar el reto de impulsar un desarrollo local y regional integral.

En la medida que haya un esfuerzo organizado de toda la sociedad local será necesario un fuerte Liderazgo que deberá asumir el gobierno local (la municipalidad). Tradicionalmente, se ha considerado a los gobiernos locales como empresas de servicios (orientadas a la ejecución de obras e infraestructuras), limitando las posibilidades de actuación de los alcaldes y funcionarios públicos en la promoción e impulso de un desarrollo integral de sus territorios. Afortunadamente, esta visión está siendo superada en la mayoría de los países que están inmersos en un proceso de descentralización territorial que, entre otros, provee a las municipalidades y gobiernos regionales de las herramientas formales necesarias para asumir adecuadamente un mayor grado de autonomía en la gestión y distribución de los recursos.

El mejoramiento de las actuales estructuras y sistemas burocráticos en los gobiernos municipales de la Macro Región Sur, se debe fortalecer Io siguiente: División técnica del trabajo administrativo, Control, monitoreo y supervisión permanente, evaluar el uso de los resultados obtenidos respecto a los cambios o impactos esperados, disponer la aplicación de medidas correctivas que contribuyan a "despersonalizar" las decisiones de políticas municipales. Los gobiernos municipales de la Macro Región Sur, deben tener en cuenta el uso adecuado de la legislación actual, inclusión de actividades programadas de capacitación, especialización y perfeccionamiento, orientar los resultados al servicio del ciudadano y la planificación debe abarcar las visiones de todos los distintos actores sociales.

Podemos concluir que la inadecuada administración de la nueva Gestión Pública influye de una manera significativa en la aplicación de los indicadores de gestión de las Municipalidades Provinciales. Las estructuras políticas y administrativas de los gobiernos municipales en la Macro región Sur son inadecuadas. Los indicadores de gestión de los gobiernos municipales en el Perú se caracterizan por tener fuerte orientación hacia la ejecución de obras y desarrollo de la infraestructura, limitando el impulso de un desarrollo integral que favorezcan el crecimiento económico, la democracia política, el progreso social, y el desarrollo humano sostenible.

\section{BIBLIOGRAFIA}

Aibar, G. (2003). Los indicadores de gestión en las entidades públicas: Implicaciones para los sistemas de información. Partida Doble, 13(147), 74-83. 
Armijo, M. (2011). Planes Nacionales de Desarrollo en países de América Latina y el Caribe: análisis preliminar de sus propuestas. ILPES. Versión preliminar.

Castillo, J. (2004a). Reingeniería y gestión municipal. Universidad Nacional de San Marcos. Lima.

Castillo, J. (2004b). Reingeniería y gestión municipal. Universidad Nacional de San Marcos. Lima.

Díaz, D. \& Xifrá, J. V. (2005). Cálculo de costes e indicadores de gestión en los servicios municipales. Editorial Civitas.

Encinas O. F. (2014). Orientación de las organizaciones públicas al aprendizaje organizacional", Revista Estudios Gerenciales, Elsevier Doyma.

Erkizia O., Agustín. (2012). Bases metodológicas para el diseño y desarrollo de un modelo de indicadores para la mejora de la gestión de los servicios públicos locales mediante la utilización de la técnica del benchmarking: Un estudio empírico.

Franciskovic, J. (2013). Retos de la gestión pública, presupuesto por resultados y rendición de cuentas", Universidad Esan, artículo publicado en el Journal of economics, finance and administrative science.
González I. \& M., Ricardo. (2010). Del síndrome del casillero vacío al desarrollo inclusivo: buscando los determinantes de la distribución del ingreso en América latina". Documento de trabajo del área de políticas presupuestarias y gestión pública, ILPES, CEPAL, Naciones Unidas. Santiago.

Hintze, J. (2003). Instrumentos de evaluación de la gestión del valor público. In ponencia presentada para el VIII Congreso Internacional del CLAD sobre la Reforma del Estado y la Administración Pública. Panamá (pp. 28-31).

Matei, A., \& Antonie, C. (2014). The New Public Management within the Complexity Model. ProcediaSocial and Behavioral Sciences, 109, 1125-1129.

Mendighttti C. A. (2012). Estrategia de la Tecnología de Información en los Gobiernos Locales", Tesis Doctoral. Lima, Perú.

Pérez L. \& Ortiz D. (2011). La implantación de medidas de gestión en la administración local: una aproximación". XVI Congreso AECA, pp. 1-22.

Torres, L. (2002). La Auditoria operativa un instrumento para la mejora de la nueva gestión pública. Revista AECA, (61).

Recibidos: 28 / 11 / 16

Aceptado para publicación: 9/ 6 / 17 